\title{
Acquisition and Database Management System for the Endoscopic Images
}

\author{
Dr H N Suma \\ Professor \& Head \\ Department of Medical \\ Electronics, B M S College of \\ Engineering, Bangalore, \\ Karnataka, India
}

\author{
Appaji M Abhishek \\ Assistant Professor \\ Department of Medical \\ Electronics, B M S College of \\ Engineering, Bangalore, \\ Karnataka, India
}

\author{
Soni Ashish Kumar \\ Department of Medical \\ Electronics, B M S College of \\ Engineering, Bangalore, \\ Karnataka, India
}

\begin{abstract}
High incidence of abdominal cancer cases in Asia has triggered a need to develop an effective automated classification of Endoscopy images based on stomach abnormalities. Currently a computer-based search for endoscopic image is not employed. The only method that is used to search an image from large image Database is manual. The images are searched one by one by the doctor amounting to lot of expert time and also may lead to subjective and probable erroneous decisions.

The proposal is therefore made to develop a new technique for automated analysis and search of the endoscopic images based on stomach abnormalities. The proposed method involves creating and maintaining Relational Database Management System (RDBMS) for endoscopic image. It is used to classify endoscopic images to normal and potentially abnormal categories. This work proposes an automated way to differentiate between the images quickly. In this method the database is set by user i.e. doctor or any technician. The proposed technique then differentiates the abnormal image from the normal and then categorized into Tumor, Ulcer, Cancer or Polyp as individual classes.

A computer-assisted method for automated endoscopic image search and analysis has been proposed. Initial results suggest the feasibility of the proposed method for stomach abnormalities. The proposed new computer-assisted endoscopic analysis method is devised to be beneficial for automatic classification of the images.
\end{abstract}

\section{General Terms}

Database Management System, Endoscopy.

\section{Keywords}

Abdominal abnormalities, Image Classification, Image Analysis.

\section{INTRODUCTION}

The first endoscope, of a kind, was developed in 1806 by Philipp Bozzini in Mainz with his introduction of a "Lichtleiter" (light conductor) "for the examinations of the canals and cavities of the human body". However, the Vienna Medical Society disapproved of such curiosity. An endoscope was first introduced into a human in 1822 by William Beaumont, an army surgeon at Mackinac Island Michigan. The use of electric light was a major step in the improvement of endoscopy. The first such lights were external. Later, smaller bulbs became available making internal light possible, for instance in a hysteroscope by Charles David in 1908. Hans Christian Jacobaeus has been given credit for early endoscopic explorations of the abdomen and the thorax with Laparoscopy (1912) and Thoracoscopy (1910). Laparoscopy was used in the diagnosis of liver and gallbladder disease by Heinz kalk in the 1930s. Hope reported in 1937 on the use of laparoscopy to diagnose ectopic pregnancy. In 1944, Raoul Palmer placed his patients in the Trendelenburg Position after gaseous distension of the abdomen and thus was able to reliably perform gynaecologic laparoscopy [1].

\section{PROPOSAL}

A pinnacle device is proposed that acquires the image and save the image into the hard disc. This device converts an analog signal into digital signal. It runs in any operating system like $\mathrm{Xp}$, Linux and vista.

First the device was tested on arthroscopy. It was working successfully. The inability to get the images for processing, because of fewer patients undergoing arthroscopic surgery, the same device is tried with endoscopy. The images are acquired for the analysis through the recording system.

\subsection{Recording System}

A video capture card is a computer component that allows camera signals to be received by a computer and allowing them to record real-time surgery onto a hard disk. Analogue Camera output a raw video stream, suitable for real-time viewing but ideally requiring some sort of compression if it is to be recorded. More advanced video capture card encode the signal to Motion JPEG or MPEG, relieving the main CPU of this load. Some cards also have analog input (S-Video/Composite input).

\subsection{Pinnacle Studio (Recording Device):}

Pinnacle studio video capture card is a custom integrated circuit developed by Pinnacle providing professional video solution to record and save real-time surgery videos. Both the Pinnacle Studio hardware and v.11 software were developed by Pinnacle. So they are designed to work together, ensuring compatibility and the highest performance. With the provided, high-speed USB 2.0 device captures video from any analog or digital source. The process is to connect and simply capture video from analogue camera output which has the format as, 
- Video capture in MPEG-1, MPEG-2 or AVI format.

- $\quad$ S-Video/Composite input.

- DV/FireWire input and output.

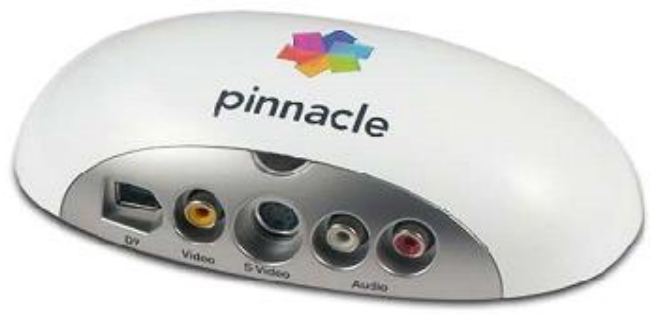

Figure 1: Pinnacle video capturing card.

\section{DATABASE MANAGEMENT SYSTEM}

A database (DB) is an organized collection of data for one or more uses, typically in digital form. The data are typically organized to model a certain aspect of reality, an application (e.g., the manufacturing processes in the company X) in a way that the views reflected by such a collection can help to follow and manage these aspects/application. The term "database" refers both to the way its users view it, and to the logical and physical materialization of its data, content, in files, computer memory, and computer data storage [2].

A DB is different from a Database management system (DBMS) which allows changing and storing the $\mathrm{DB}$, as well as retrieving information from it. Such information is computed (by the DBMS) from the DB's stored data. A specific DB with specific data is the vehicle to manage a specific application, while the DBMS is the tool to use and maintain the DB. DBMSs are packaged as computer software products. Each DBMS type can support a DB type which is specific to the DBMS, as designed by the DBMS manufacturer/developer(s). Several popular general-purpose DBMS types exist, like the Oracle DBMS, SQL Server of Microsoft, DB2 of IBM and the Open source DBMS MySQL.

Each such DBMS type currently supports many thousands of DBs all over the world. DBs are not portable across different DBMS, but can inter-operate to some degree (while each DBMS type controls a DB of its own DB type) using standards like SQL and ODBC. A successful general-purpose DBMS is designed in a way that is can satisfy as many as possible different applications and application designers and builders. A DBMS also needs to provide effective run-time execution to properly support (e.g., in terms of performance, availability, and security) as many end-users (the DB's application users) as needed. Sometimes the combination of a DB and its respective DBMS is referred to as a Database system (DBS).

\subsection{Database management system consists of following technology:}

- $\quad$ HTML as front end (user side).

- JavaScript client-side language.

- $\quad$ PHP for server-side scripting language.

- MySQL for relational database management system (RDBMS).
- XAMPP open server solution stack package [19].

\subsection{HTML}

HTML which stands for Hyper Text Mark up Language is the predominant mark up language for web pages. HTML is not a programming language, it is a mark up language. HTML is the basic building-block of webpages. HTML is written in the form of HTML elements consisting of tags, enclosed in angle brackets (like $<\mathrm{html}>$ ), within the web page content. HTML tags normally come in pairs like $\langle\mathrm{h} 1\rangle$ and $\langle/ \mathrm{h} 1\rangle$. The first tag in a pair is the start tag, the second tag is the end tag (they are also called opening tags and closing tags).HTML also allows images and objects to be embedded and can be used to create interactive forms. It provides a means to create structured documents by denoting structural semantics for text such as headings, paragraphs, lists, links, quotes and other items. It can embed scripts in languages such as JavaScript which affect the behaviour of HTML pages [3].

\author{
Syntax: \\ $<\mathrm{Html}>$ \\ $<$ body $>$ \\ $<$ h1 $>$ MyFirstHeading $\langle/$ h1 $>$ \\ $<$ p $>$ Myfirstparagraph $</ \mathrm{p}>$ \\ $</$ body $>$ \\ $</ \mathrm{Html}>$
}

\subsection{JavaScript}

JavaScript, also known as ECMA Script is a prototype-based, object-oriented scripting language that is dynamic, weakly typed and has first-class functions. It is also considered a functional programming language like Scheme and OCaml because it has closures and supports higher-order functions.

JavaScript is an implementation of the ECMA Script language standard and is primarily used in the form of client-side JavaScript, implemented as part of a web browser in order to provide enhanced user interfaces and dynamic websites. This enables programmatic access to computational objects within a host environment.

JavaScript uses syntax influenced by that of C. JavaScript copies many names and naming conventions from Java, but the two languages are otherwise unrelated and have very different semantics. The key design principles within JavaScript are taken from the self and Scheme programming languages.

\subsection{PHP}

PHP is a general - purpose scripting language originally designed for web development to produce dynamic web pages. For this purpose, PHP code is embedded into the HTML source document and interpreted by a web server with a PHP processor module, which generates the web page document. It also has evolved to include a command-line interface capability and can be used in standalone graphical applications. PHP can be deployed on most web servers and as a standalone interpreter, on almost every operating system and platform free of charge. 
PHP is installed on more than 20 million websites and 1 million web servers [4].

Features of PHP are:

- PHP runs on different platforms (Windows, Linux, UNIX, etc.)

- $\quad$ PHP is compatible with almost all servers used today (Apache, IIS, etc.)

- $\quad$ PHP is a server-side scripting language, like ASP.

- PHP supports many databases (MySQL, Informix, Oracle, Sybase, Solid, PostgreSQL, Generic ODBC, etc.)

- $\quad$ PHP is open source software.

- $\quad$ PHP is free to download and use.

\subsection{MYSQL}

MySQL is a relational database management system (RDBMS) that runs as a server providing multi-user access to a number of databases. The SQL phrase stands for Structured Query Language.

The MySQL development project has made its source code available under the terms of the GNU General Public License, as well as under a variety of proprietary agreements. MySQL was owned and sponsored by a single for-profit firm, the Swedish company MySQL AB, now owned by Oracle Corporation.

Free-software-open source projects that require a full-featured database management system often use MySQL. It is also used in many high-profile, large-scale World Wide Web products, including Wikipedia, Google (though not for searches) and Facebook.

MYSQL features are:-

- MySQL is a database server

- MySQL is ideal for both small and large applications

- MySQL supports standard SQL

- MySQL compiles on a number of platforms

- MySQL is free to download and use

\subsection{XАMPP}

XAMPP is a free and open source cross-platform web server solution stack package, consisting mainly of the Apache HTTP Server, MySQL database, and interpreters for scripts written in the PHP and Perl programming languages.

XAMPP's name is an acronym for:

- $\quad \mathrm{X}$ (meaning cross-platform)

- $\quad$ Apache HTTP Server

- MySQL

- $\quad$ PHP

- Perl

The program is released under the terms of the GNU General Public License and acts as a free web server capable of serving dynamic pages. XAMPP is available for Microsoft Windows, Linux, Solaris, and Mac OS X, and is mainly used for web development projects. This software is useful while you are creating dynamic webpages using programming languages like
PHP, JSP, Servlets. XAMPP also provides support for creating and manipulating databases in MySQL and SQLite among others. Once XAMPP is installed you can treat your local host like a remote host by connecting using an FTP client.

\subsection{Apache HTTP Server}

The Apache HTTP Server, commonly referred to as Apache is web server software notable for playing a key role in the initial growth of the World Wide Web. In 2009 it became the first web server software to surpass the 100 million website milestone. Apache was the first viable alternative to the Netscape Communications Corporation web server (currently known as Oracle iPlanet Web Server), and has since evolved to rival other web servers in terms of functionality and performance. Typically Apache is run on a Unix-like operating system.

Apache is developed and maintained by an open community of developers under the auspices of the Apache Software Foundation. The application is available for a wide variety of operating systems, including UNIX, GNU, FreeBSD, Linux, Solaris, Novell NetWare, AmigaOS, Mac OS X, Microsoft Windows, OS/2, TPF, and eComStation. Released under the Apache License, Apache is characterized as open-source software.

\section{Block Diagram}

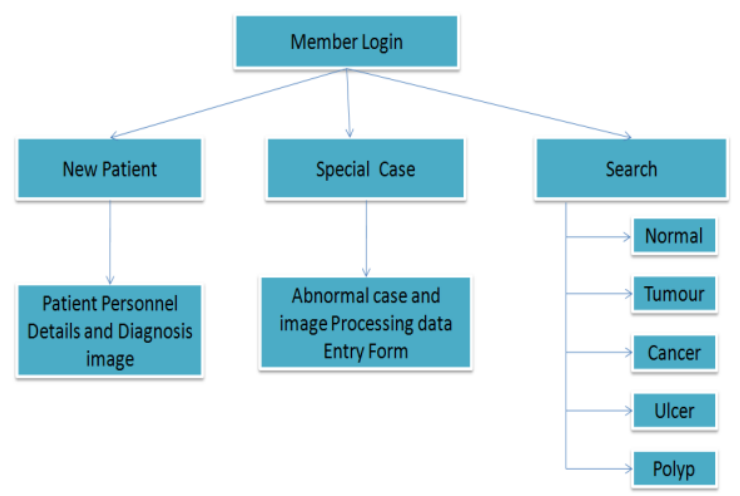

Figure 2: Database management of endoscopic images.

The above block diagram explains that how database management of endoscopic images is created and maintained for every patient. The image of patient with personnel details is stored in database for future analysis and research. This database is created for an automated search of the normal and abnormal image case like Tumour, Cancer, Ulcer and Polyp. There is smart search option which display all image of special case in single window.

\section{DECISION MAKING}

For all the images the maximum and the minimum values is obtained and a rule based method is applied for the classification [5]. 


\subsection{Block diagram of Rule Based Method}

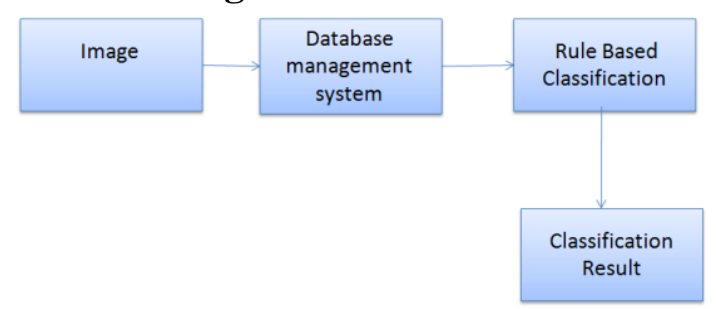

Fig 3: Block diagram of Rule based method.

Rule-based theories of concept learning take classification data and a rule-based theory as input, which are the result of a rulebased learner with the hopes of producing a more accurate model of the data. The majority of rule-based models that have been developed are heuristic, meaning that rational analyses have not been provided and the models are not related to statistical approaches to induction. A rational analysis for rulebased models could presume that concepts are represented as rules, and would then ask what degree of belief a rational agent should be in agreement with each rule, provided some observed example. Rule based theories of concept learning are focused more so on perceptual learning and less on definition learning [6]. Rules can be used in learning when the stimuli are confusable as opposed to simple. When rules are used in learning, the decisions are made based on properties alone and rely on simple criteria that do require a lot of memory.

Example of Rule based theory:

"A radiologist using rule-based categorization would observe whether specific properties of the X-ray meet certain criteria; for example, is there an extreme difference in brightness in a suspicious region relative to the other regions? A decision is then based on this property alone".

\subsection{Goal:}

The rule-based classification is use to classify Endoscopic images. Here we aim to minimize confusion errors between spectrally similar classes. The strategy for discriminating between normal and abnormal is to label all images beyond certain value as normal and other as the abnormal. Object classification is then used to differentiate between images.

\subsection{Statistical Analysis:}

The attributes used are the mean, median, RMS value and the entropy of the segmented image. Since the image has already been classified using maximum likelihood classification in a visual approach, image segments can be initially assigned by analyzing classes of images belonging to every segment.

After getting all these data a certain range is given for all the cases and if- else condition is applied so that if any new images occurred and if it satisfy the condition it will be classified as that category.

\section{RESULTS}

Screen shots of Database management System of Endoscopic Department in figure 4 to 11.

\section{CONCLUSION}

The Database management system can be applied successfully to endoscopic images. New approaches for search and analysis of the stomach abnormality for endoscopic images have been developed. Classification using Decision making are important features used which are able to distinguish the normal and abnormal image and also were able to classify the images into four different categories.

Overall, the process showed convincing results and achieved a fast and higher accuracy with respect to manual search. Further work will go into optimization of the system and into development of further modules to aid the surgeon. It is believe that Database management system will play an increasingly important role in endoscopic image analysis and future studies or research of cases.

This paper has been done to fulfil most of the basic needs to investigate the implementation of this algorithm in real time application. Here we have taken care to deal with real time data like Endoscopic image, but still it can be modified to enhance its features which could not be included due to time constraints and in depth knowledge of XAMPP Server and PHP. The salient features that might be further added are as follows:

- The tool can be made more user friendly making it complete GUI.

- Uploading image directly from source folder make it works faster in a real time data entry.

- Handling of large file due to insufficient memory in local disk i.e. $\mathrm{C}$ drive is a problem.

- Uploading image above $500 \mathrm{~kb}$ size.

- Mysql can be directly connected with Matlab. 


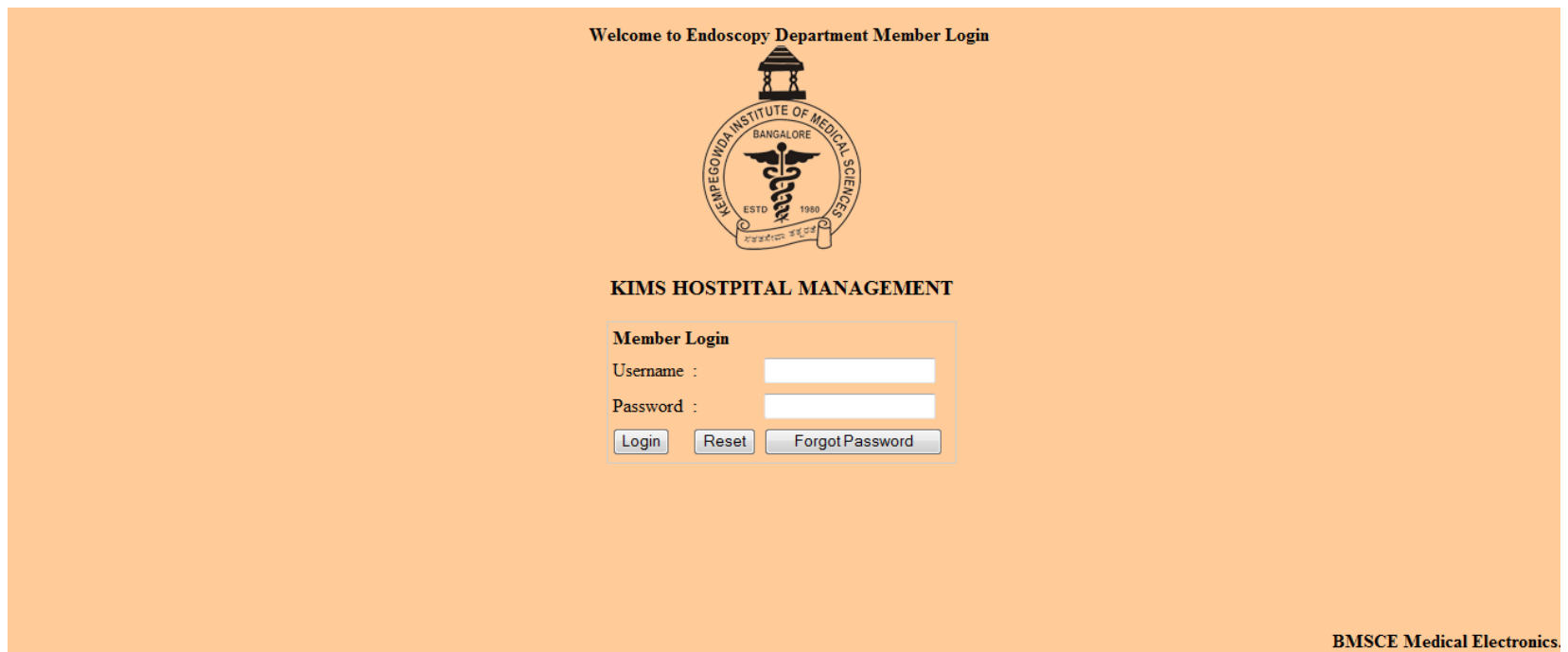

Fig 4: Member Login Page

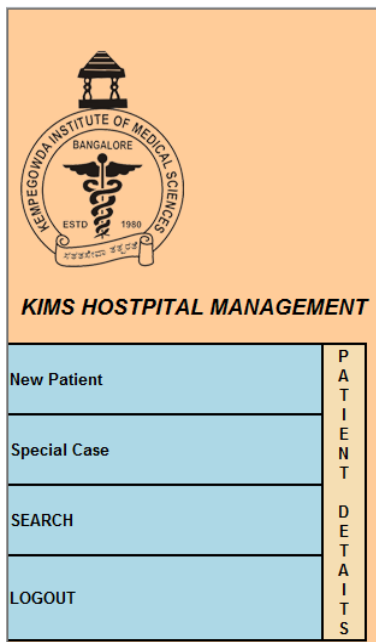

Welcome to Endoscopy Department Home Page

Fig 5: Endoscopy Home page

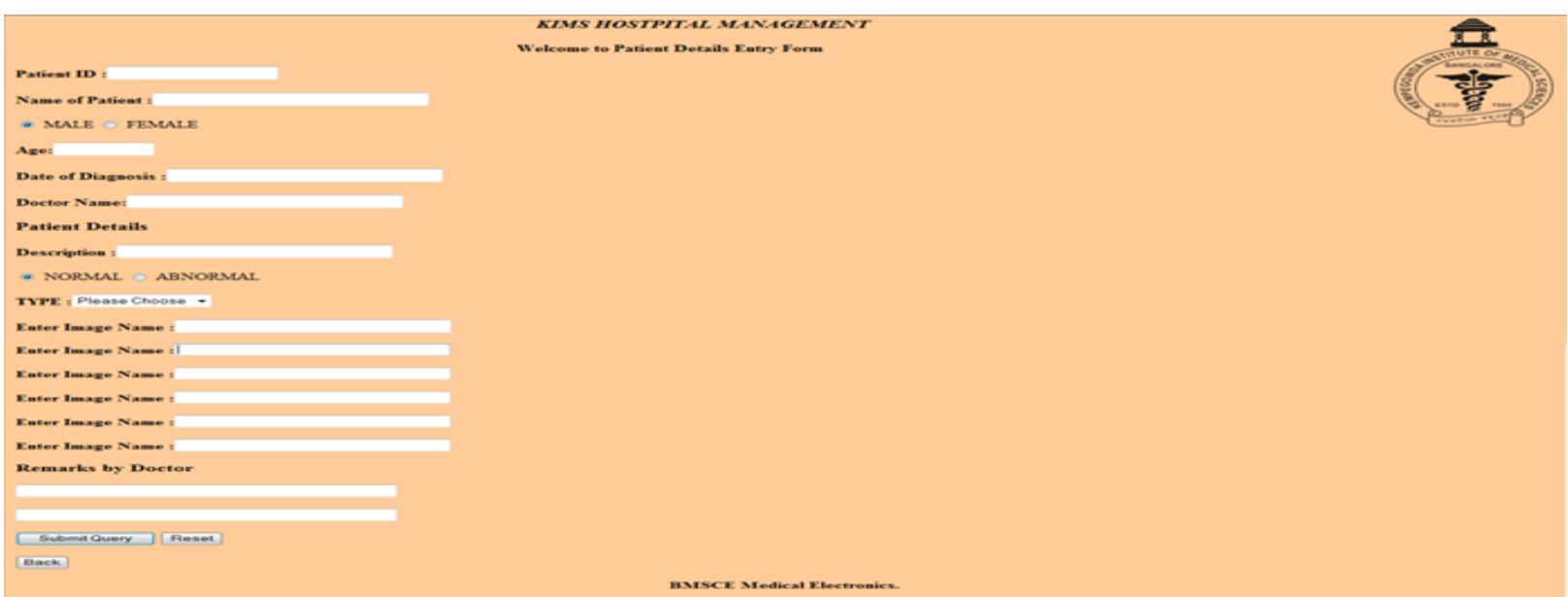

Fig 6: New Patient entry Form 


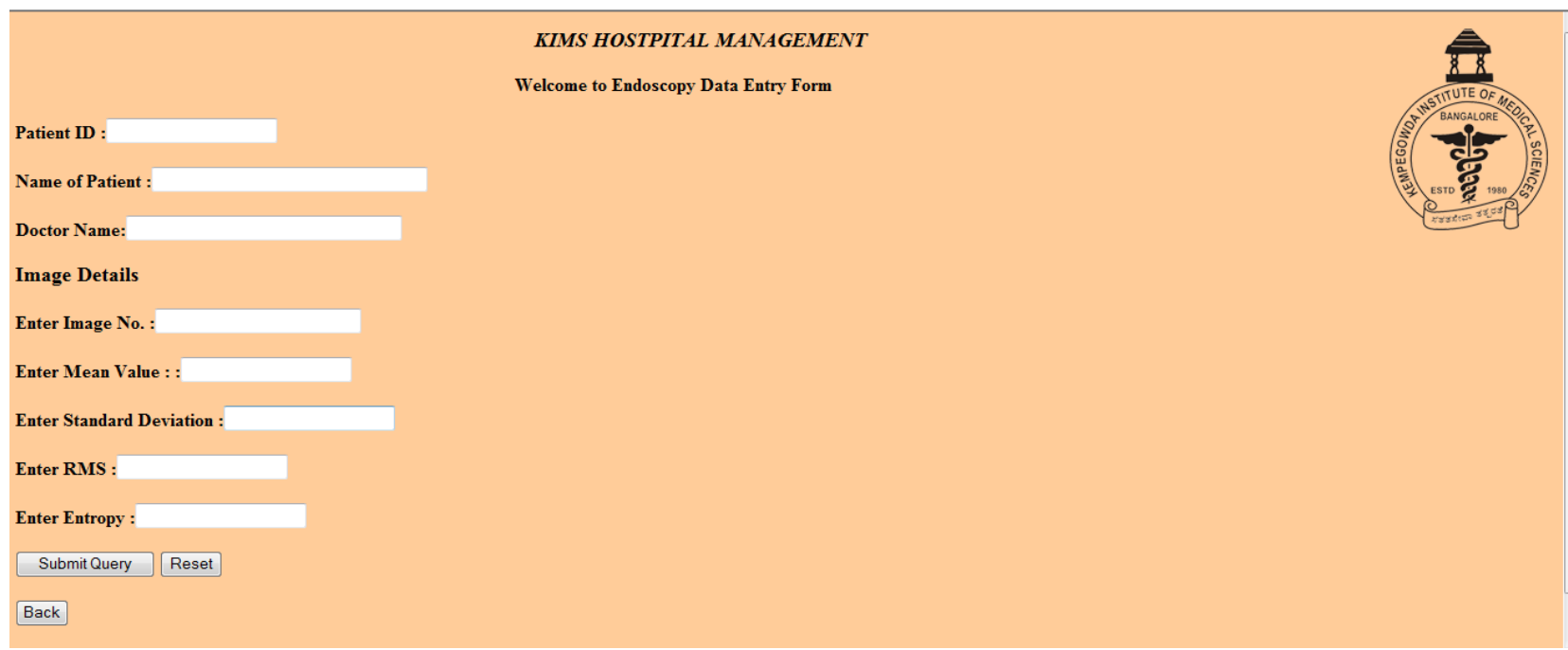

Fig 7: Special case entry Form

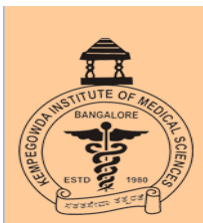

KIMS HOSTPITAL MANAGEMENT

\begin{tabular}{|c|}
\hline HOME \\
\hline TUMOUR \\
\hline CANCER \\
\hline ULCER \\
\hline POLYP \\
\hline NORMAL \\
\hline
\end{tabular}

Endoscopy Image Search Option

\section{Fig 8: Image search option}

BMSCE Medical Electronics

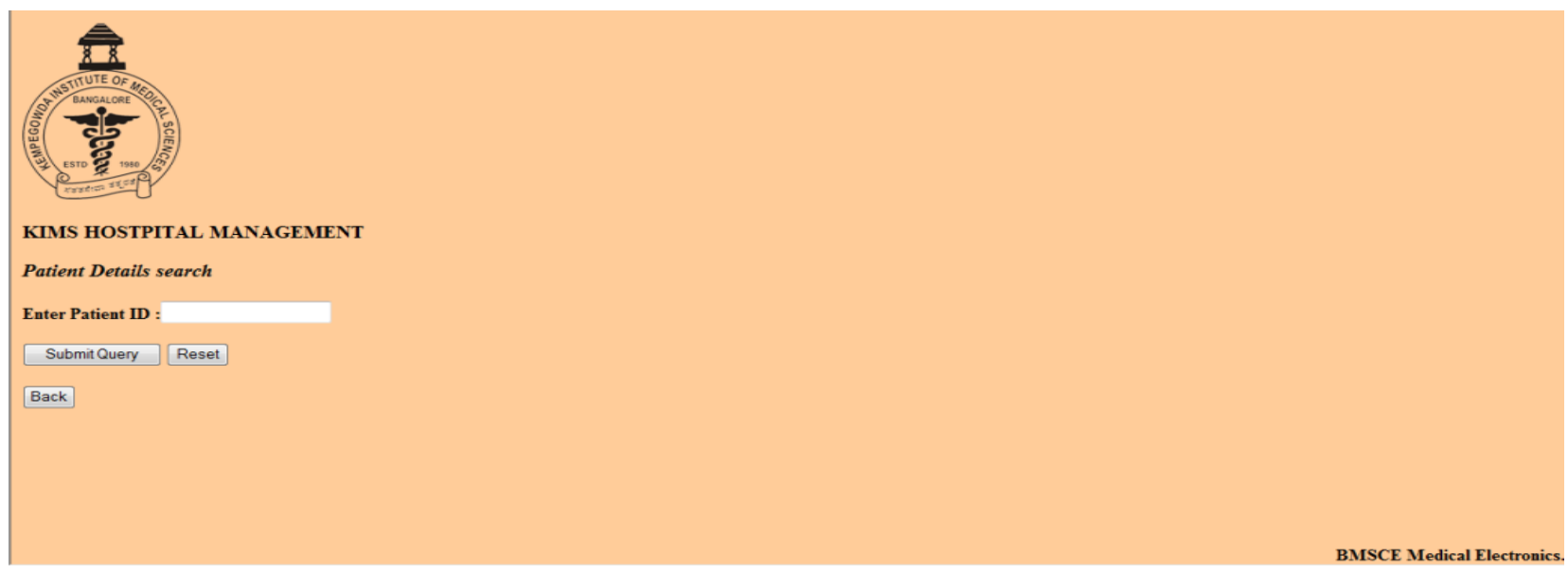

Fig 9: Patient Details search entry form 


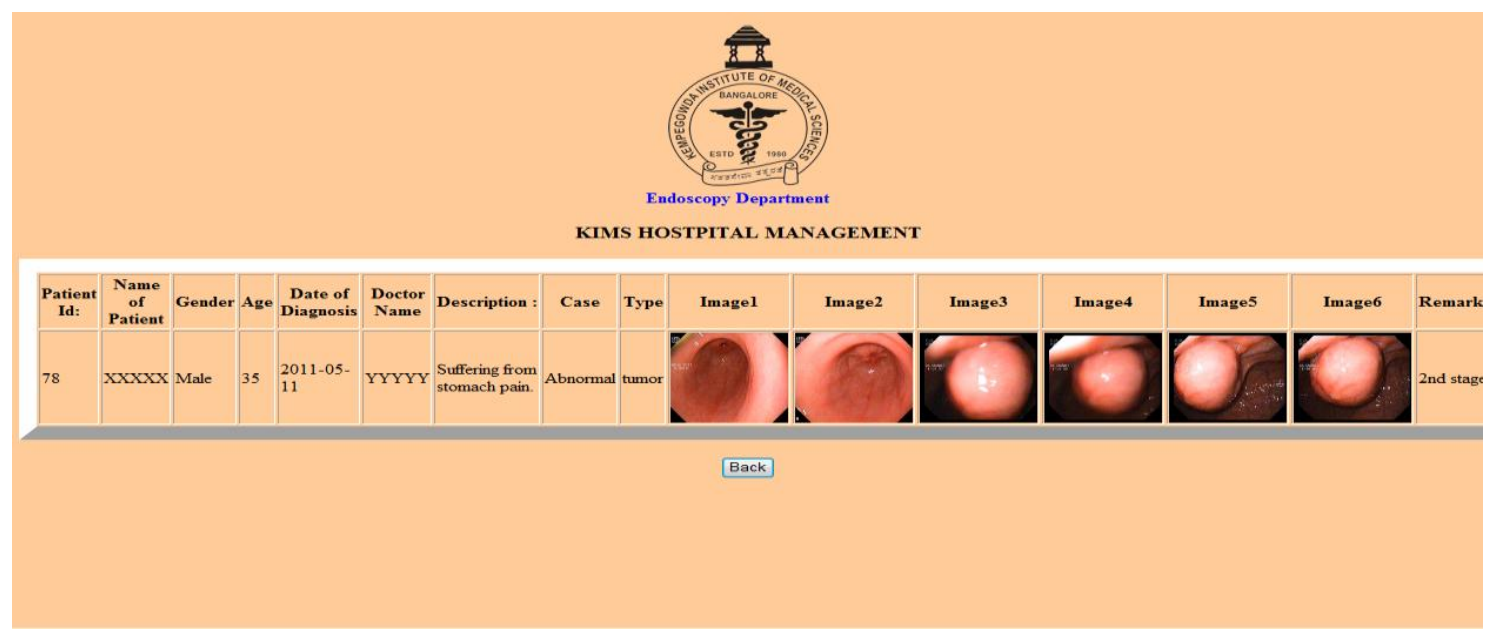

Fig 10: Patient search result fetch from database

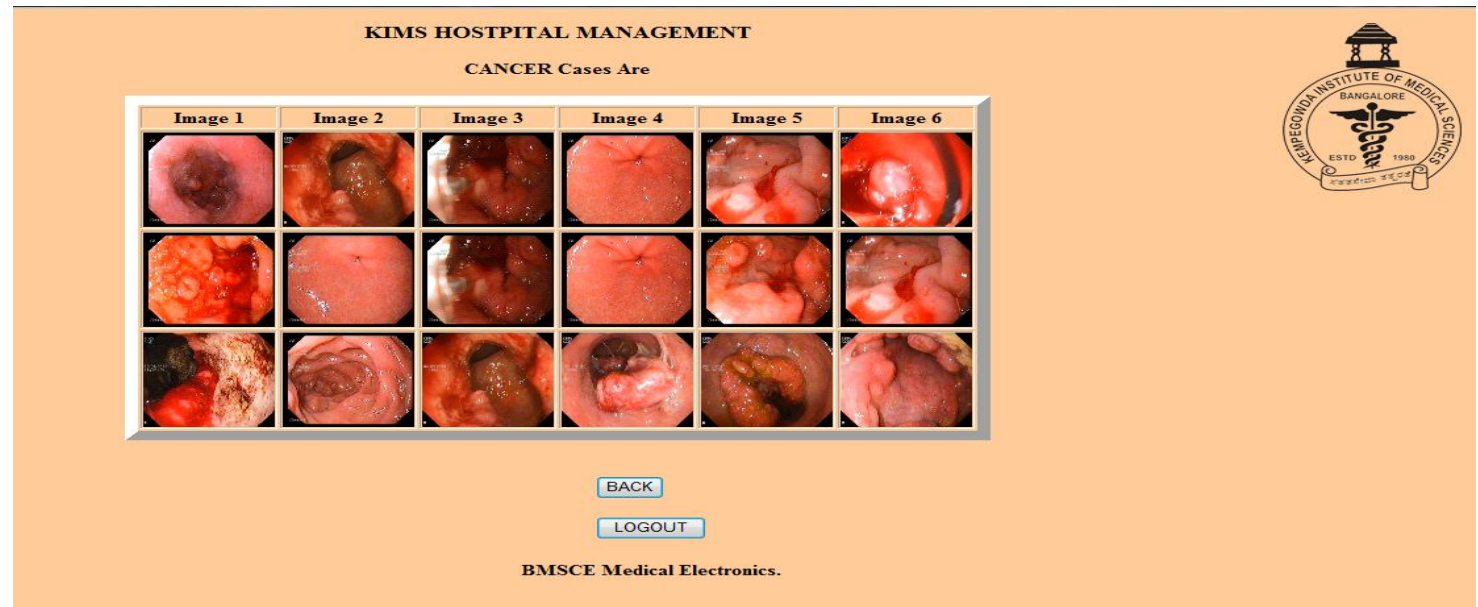

Fig 11: Cancer case search from database

\section{ACKNOWLEDGMENT}

We acknowledge and extend warm regards to our guide Dr. R. Sahadev, Associate Professor, Department of Surgical Gastroenterology, Kempegowda Institute of Medical Sciences, Bangalore, Karnataka for all his assistance guidance and time in bringing this paper to completion. A special thanks to Dr. N.C Srinivasa Prabhu, Professor, Department of Medicine, Kempegowda Institute of Medical Science, Bangalore, for his valuable and timely support.

\section{REFERENCE}

[1] G.N. Khan and D.F. Gillies, "Vision based navigation system for an endo-scope," Image and Vision computing, vol. 14, pp. 763-772, 1996.

[2] Altekruse SF, Kosary CL, Krapcho M, et al (eds). SEER Cancer Statistics Review, 1975-2007,National Cancer Institute. Bethesda, MD, http://seer.cancer.gov/csr/1975_2007/, based on November 2009 SEER data submission, posted to the SEER web site, 2010.

[3] Soekhoe JK, Groenen MJM, van Ginneken AM, et al. Computerized endoscopic reporting is no more time consuming than reporting with conventional methods. Eur J Intern Med 2007.

[4] World-Wide Computer Communications of the ACM, February 1997, Vol. 40 No 2 and the web: Past, Present and Future (1996) by Tim Berners-Lee.

[5] Network Communication Design HTML Design Guide Version 5.0 - PDF Edition 1994-2000.

[6] OBJECT-ORIENTED PHP Concepts, Techniques, and Code by Peter Lavin.

[7] A Rule-Based Method for Automated Footprint Localization and Classi_cation of Small Species Haokun Geng, Cheryl Mills, Radu Nicolescu, and Reinhard Klette Department of Computer Science, University of Auckland, Private Bag 92019Auckland, New Zealand.

[8] Rule-Based Classification of a Very High Resolution Image in an Urban Environment Using Multispectral Segmentation Guided by Cartographic Data Mourad Bouziani, Kalifa Goita, and Dong-Chen He,IEEE Transactions On Geoscience And Remote Sensing, Vol. 48, No. 8, August 2010. 